\title{
Malaria treatment in remote areas of Mali: use of modern and traditional medicines, patient outcome
}

\section{Drissa Diallo ${ }^{a}$, Bertrand $\mathrm{Graz}^{\mathrm{b}, *}$, Jacques Falquet ${ }^{\mathrm{b}}$, Abdel Kader Traoréc, $^{c}$ Sergio Giani ${ }^{\mathrm{d}}$, Pakuy Pierre Mounkoro ${ }^{\mathrm{a}}$, Adama Berthée, Massambou Sackof ${ }^{\text {, Chiaka Diakité }}{ }^{a}$}

\author{
a Département de Médecine Traditionnelle (DMT), Bamako, Mali \\ b Antenna Technologies, Geneva, Switzerland \\ c Centre National de la Lutte Contre la Maladie (CNAM), Bamako, Mali \\ d Aide au Développement des Médecines traditionnelles (AIDEMET), Bamako, Mali \\ e Direction de la Pharmacie et du Médicament, Bamako, Mali \\ f Direction Nationale de la Santé: Programme National de Lutte contre le Paludisme, Bamako, Mali
}

Received 5 April 2005; received in revised form 17 August 2005; accepted 17 August 2005

Available online 17 October 2005

\section{KEYWORDS \\ Malaria; \\ Rural health; \\ Traditional \\ medicines; \\ Medicinal plants; \\ Public health; \\ Mali}

Summary Use of official health services often remains low despite great efforts to improve quality of care. Are informal treatments responsible for keeping a number of patients away from standard care, and if so, why? Through a questionnaire survey with proportional cluster samples, we studied the case histories of 952 children in Bandiagara and Sikasso areas of Mali. Most children with reported uncomplicated malaria were first treated at home $(87 \%)$ with modern medicines alone (40\%), a mixture of modern and traditional treatments (33\%), or traditional treatment alone (27\%). For severe episodes (224 cases), a traditional treatment alone was used in $50 \%$ of the cases. Clinical recovery after uncomplicated malaria was above $98 \%$ with any type of treatment. For presumed severe malaria, the global mortality rate was $17 \%$; it was not correlated with the type of treatment used (traditional or modern, at home or elsewhere). In the study areas, informal treatments divert a high proportion of patients away from official health services. Patients' experience that outcome after standard therapeutic itineraries is not better than after alternative care may help to explain low use of official health services. We need to study whether some traditional treatments available in remote villages should be considered real, recommendable first aid.

(c) 2005 Royal Society of Tropical Medicine and Hygiene. Published by Elsevier Ltd. All rights reserved.

\footnotetext{
* Corresponding author. Present address: c/o le Château, Galland, 1374 Corcelles-sur-Chavornay, Switzerland.

E-mail address: bertrand.graz@chuv.ch (B. Graz).
} 


\section{Introduction}

In Mali, attendance at community health centres often remains poor despite great efforts to improve quality of care. Several explanations have been proposed: patients' lack of resources, long distances from home to the nearest health centre, poor health services management (Huissoud et al., 2004). This situation seems common in Africa, and has been related to the fact that modern medicines often remain problematic in remote areas: even when apparently available, they are often unaffordable, misused or even counterfeit (Basco, 2004; Dossou-Yovo et al., 2001; Tumwesigire and Watson, 2002).

From discussions with community representatives, we suspected that another reason could partly explain low attendance in community health centres: self-medication and traditional medicines might keep patients away from official health services. We were able to test this hypothesis through a case study: an enquiry about therapeutic practices for children with presumed malaria, uncomplicated or severe, in remote rural areas.

\section{Methods}

\subsection{Study design}

This work was a collaborative programme between the Department of Traditional Medicine (National Institute of Research in Public Health, Mali) and Antenna Technologies (Geneva, Switzerland). A population-based retrospective survey, with questionnaires, was conducted in two rural districts in southern and eastern Mali. Field research was complemented by laboratory screening of the local treatments correlated with the best clinical outcome.

\subsection{Definition of malaria}

Two perspectives were used and compared: popular knowledge, which is the basis for therapeutic decisions at the village level, and community health centre clinical definitions. It was already known that in Mali popular knowledge distinguishes between uncomplicated and severe malaria (Traoré, 1999). The clinical definition of malaria was based on national malaria policy; in community health centres, where laboratory tests are not available, any fever is considered to be malaria unless there is another obvious cause and any fever with coma or convulsion during the rainy season or soon after is treated as severe malaria. Recent studies tend to increase reliance on clinical definitions of malaria, in showing that Plasmodium falciparum parasitaemia is not well correlated with actual disease (some patients have low parasitaemia and severe symptoms; others have high parasitaemia with no clinical disease) except for very high parasitaemias (Delley et al., 2000; Von Seidlein et al., 2002).

\subsection{Study sites}

The study areas were chosen in view of their relative isolation (long distances to the modern health centre). The health area of Kendié is located in Bandiagara region, $45 \mathrm{~km}$ away from the nearest city and hospital of Bandiagara. The vast majority of inhabitants are farmers. In Kendié there is a community health centre and there is also a traditional healers' association. The health area of Finkolo AC is situated in Sikasso region, $40 \mathrm{~km}$ away from the region's capital, Sikasso, on the road to Burkina Faso. In Finkolo there is a community health centre. In the area there are many traditional healers, who are not organized in an association. Agriculture is the main economic activity in this region.

\subsection{Population}

A questionnaire was administered in households at the end of the rainy season (high transmission period). As it is usually impossible to have just one person answering a questionnaire in a village, every interview was set up as a mini-focus group (with the mother, and a few family members) about one particular patient (age $0-16$ years) and disease episode with a recall period of 2 weeks for an uncomplicated episode and 6 months for severe malaria. Proportional cluster samples were drawn according to the population census and continuous civil records of the areas (Bennett et al., 1991). Sample size was decided on the basis of the expected prevalence of malaria in the area, in order to find at least 600 uncomplicated malaria cases.

\subsection{Data collection}

As this study was, to our knowledge, the first of its kind (i.e. with this attention to outcome and disease severity), we had to construct and validate an original questionnaire. Several drafts were revised by malaria researchers, public health specialists, pharmacists, anthropologists, local health workers, malaria patients and parents. Then the questionnaire was pre-tested in 34 houses, with similar ethnic composition, adjacent to the study areas. Data were collected in December 2002-January 2003.

\subsection{Data analyses}

Data were analysed with Epilnfo 6 software (CDC, Atlanta, GA, USA), with $\chi^{2}$ and Fisher's exact tests for discrete variables, and Kruskall-Wallis test for means.

\subsection{Laboratory analyses}

Samples of plants with the best clinical outcomes, determined by analysis of correlation between outcome and treatments (Graz et al., 2005) were collected in the Sikasso region. The plant parts used locally (e.g. stems and leaves) were extracted with water and organic solvents (dichloromethane, methanol and ethanol) at the Department of Traditional Medicine in Bamako. Extracts were freeze-dried and used for biological tests.

The extracts were tested for their $\mathrm{IC}_{50}$ (concentration inhibiting $50 \%$ of parasite growth) on cultured chloroquineresistant $P$. falciparum (strain K1, stage IEF, Swiss Tropical Institute, Basel, Switzerland). In these tests, the control was chloroquine. 


\subsection{Ethical aspects}

The study was accepted by the Malian ethical committee. It was conducted in retrospect because we were also interested in severe malaria and its actual local treatments.

\section{Results}

Information to the local people and respect of traditional etiquette resulted in a good acceptance of the survey: from the 483 households in the Bandiagara region and 469 households in the Sikasso region, nobody refused to answer the questionnaire, so the global response rate was $100 \%$; some questions, however, were not answered by $100 \%$ of respondents, as will be mentioned below.

Unless otherwise mentioned, results presented here are derived from pooled records from both regions (Finkolo and Kendié areas). Similarities in sample sizes and results justify such pooling.

Local designations of uncomplicated and severe malaria were in fairly close agreement with definitions based on clinical criteria and used in local dispensaries and health centres (86\% agreement in Bandiagara, 98\% in Sikasso region), the latter being used for correlation analyses below.

As shown in Figure 1, self-medication (traditional or modern medicine, see below) as first treatment was the rule for uncomplicated malaria (87\% of 719 cases), with increased recourse to traditional healers and modern health centres when a second treatment was sought. For severe malaria (224 cases with a first treatment, 84 with a second), 55\% started with self-medication, while visits to traditional healers were relatively frequent ( 32 vs. $4 \%$ for an uncomplicated episode, $P<0.001)$. Modern health centres tended to receive more demands for severe episodes (13 vs. $9 \%$ for an uncomplicated episode, $P=0.08$ ).

In the case of uncomplicated malaria, more than $60 \%$ of the patients received treatment at the first symptoms. For severe malaria, only $24 \%$ were treated before convulsions or coma.

Table 1 shows that exclusive use of traditional medicine as first treatment was more common in severe malaria. Exclusive use of traditional medicine was also the most frequent second treatment, whatever the type of malaria. The traditional medicines used were mainly medicinal plants $(91 \%)$, otherwise incantations and prayers $(4 \%)$, or others (3\%). Patients with uncomplicated malaria that were treated with medicinal plants took them orally and as a bath in $95 \%$ of the cases (fumigation $4 \%$, others $1 \%$ ). For severe malaria treated with plants, $59 \%$ of patients had baths, usually fol-
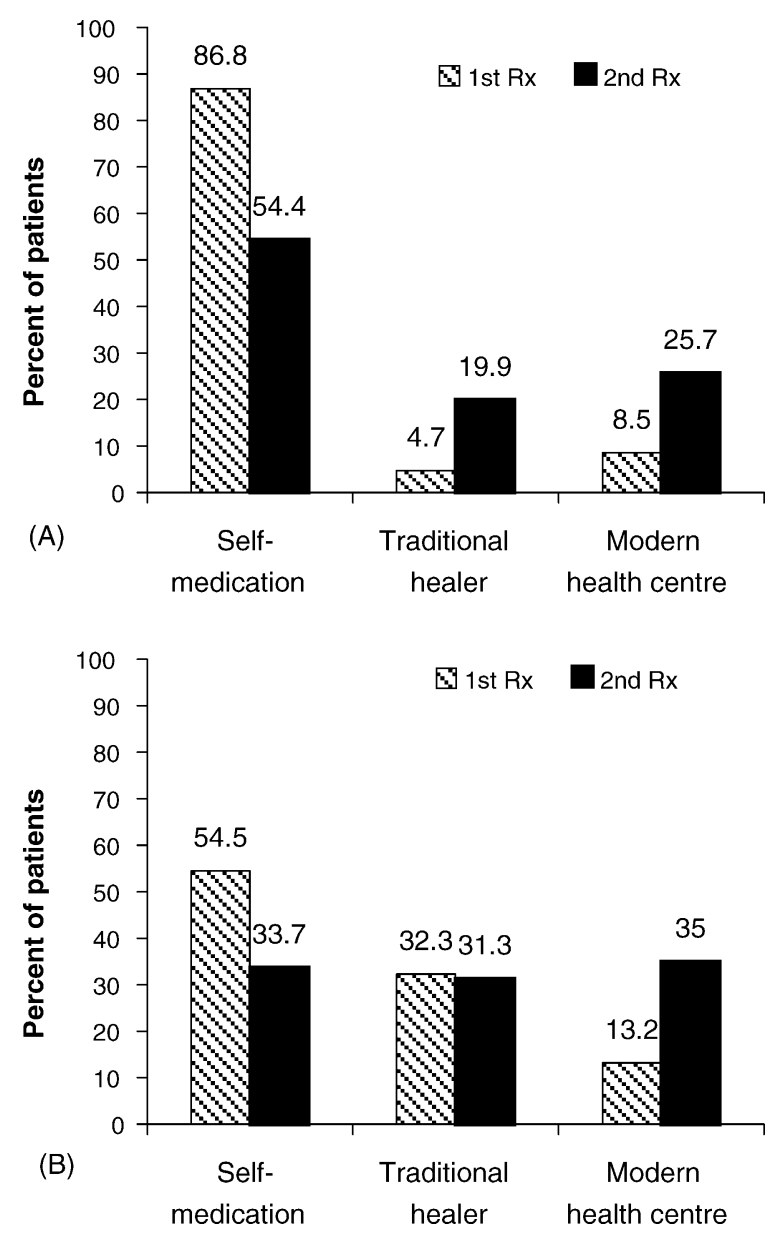

Figure 1 Health services used for first treatment (1st Rx) and second treatment ( 2 nd $\mathrm{Rx}$ ) in cases of (A) uncomplicated malaria and (B) severe malaria.

lowed by oral administration as soon as possible; fumigation and inhalation were often reported in case of severe malaria $(35 \%)$ as a way of giving treatment to a comatose or convulsing child, as well as massage with a plant-based ointment ( $4 \%$, others $2 \%)$.

The trend was towards less modern treatment with more remoteness, which was statistically significant in the case of severe malaria in the Sikasso region: among those living more than $15 \mathrm{~km}$ away from the PHC centre $(63 \%$ of the sample) more than half $(54 \%)$ started with traditional treatment only, compared with $33 \%$ among those living closer $(P=0.04)$. Modern treatment was more often sought when a motor vehicle was available in the household (alone or with

Table 1 Number and percentage of patients receiving each type of treatment

\begin{tabular}{|c|c|c|c|c|c|}
\hline & \multicolumn{2}{|c|}{ Uncomplicated malaria } & & \multicolumn{2}{|c|}{ Severe malaria } \\
\hline & 1st Rx & 2nd Rx & & 1st Rx & 2nd Rx \\
\hline Modern only, $n$ (\%) & $294(41)$ & $44(33)$ & Modern only & $54(24)$ & $29(36)$ \\
\hline Traditional only, $n$ (\%) & $183(25)$ & $88(66)$ & Traditional only & $111(50)$ & 40 (49) \\
\hline Both, $n(\%)$ & $242(34)$ & $2(1)$ & Both & $59(26)$ & $12(15)$ \\
\hline Total, n (\%) & 719 (100) & $134(100)$ & Total & $224(100)$ & $81(100)$ \\
\hline
\end{tabular}

1st Rx: first treatment; 2nd Rx: second treatment. 
Table 2 Progress of patients after different treatments

\begin{tabular}{|c|c|c|c|}
\hline Treatment & Cured & $\begin{array}{l}\text { Cured with } \\
\text { sequels }\end{array}$ & Death \\
\hline \multicolumn{4}{|c|}{ Uncomplicated malaria } \\
\hline $\begin{array}{c}\text { Modern only, } \\
n(\%)\end{array}$ & $218(100)$ & $0(0)$ & $0(0)$ \\
\hline $\begin{array}{l}\text { Traditional } \\
\text { only, } n(\%)\end{array}$ & $144(98)$ & $1(1)$ & $2(1)$ \\
\hline Both, $n(\%)$ & $326(100)$ & $0(0)$ & $0(0)$ \\
\hline Total & 688 & 1 & 2 \\
\hline \multicolumn{4}{|l|}{ Severe malaria } \\
\hline $\begin{array}{c}\text { Modern only, } \\
n(\%)\end{array}$ & $16(70)$ & $1(4)$ & $6(26)$ \\
\hline $\begin{array}{l}\text { Traditional } \\
\text { only, } n(\%)\end{array}$ & $65(82)$ & $5(6)$ & $9(11)$ \\
\hline Both, $n(\%)$ & $84(76)$ & $5(5)$ & $21(19)$ \\
\hline Total & 165 & 11 & 36 \\
\hline
\end{tabular}

traditional treatment, all cases: 73 vs. $66 \%, P=0.02$ ) and when the mother had studied in the 'madrasa' or Koranic school (85\% sought modern treatment, vs. $67 \%$ among those who had no schooling, $P=0.004)$. No relation was found between the type of treatment used and the father's profession and level of education, nor the number of other children at home. Prices of treatment (in francs CFA: 1000 CFA $\approx £ 1$ or $€ 0.8$ ) were related to type of malaria (uncomplicated episode mean price $=504$ FCFA; severe [whatever the type of treatment used] $=1340$ FCFA; $P=0.03$ ) and type of treatment (first treatment traditional mean price $=200$ FCFA; modern [whatever the type of malaria] = 830 FCFA; $P<0.001)$.

As shown in Table 2, total recovery after uncomplicated malaria was above $98 \%$ for any treatment. In the case of severe malaria, the global death rate was $17 \%$. Patient progress did not statistically differ with different types of treatment. For data missing on progress $(2.2 \%$ of uncomplicated cases, $3.6 \%$ of severe cases, $P=0.2$ ) types and places of treatment were similar to the ones found in the total sample, as well as socio-demographic data. Sequelae were reported in $5 \%$ of severe cases and $0.5 \%$ of uncomplicated cases.

None of the local treatments was correlated with poor patient progress, but some were systematically associated with clinical cure. Some of these were already known to be active against $P$. falciparum. Six plants with activities not mentioned in the scientific literature were investigated in more detail.

\subsection{In-vitro studies}

Crude extracts of the selected plants were obtained and tested in the laboratory: all were found to have medium to high antiplasmodial activity in chloroquine-resistant $P$. falciparum cultures. Four crude extracts displayed a medium inhibitory concentration $\left(\mathrm{IC}_{50}\right)$ smaller than $5 \mu \mathrm{l} / \mathrm{ml}$ : Spondias mombin, Opilia celtidifolia, Securinega virosa, Argemone mexicana.

\section{Discussion}

The objective of this study was to examine how and why use of home- or village-based treatments may keep patients away from official health services. For this, we studied therapeutic practices and subsequent patient progress in presumed malaria episodes in remote areas.

Self-medication (modern drugs or traditional medicine) was the most frequent type of first treatment sought, even for suspected severe malaria. For severe malaria, traditional medicine was the only treatment used in half the cases. After uncomplicated malaria, total recovery was above $98 \%$ with any type of treatment. For severe malaria, the global mortality rate was $17 \%$ and did not significantly differ with the type of treatment used. Some local products were systematically associated with good clinical outcome and found to have medium to high in-vitro antiplasmodial activity.

Severity in itself was not a reason for seeking modern health care: only $24 \%$ of patients with severe malaria chose modern medicine as their first option, whereas $41 \%$ of those with uncomplicated malaria did so. One reason for this could be that severe malaria is more often related to supernatural events (Berche, 1994). Another explanation could be the perceived effectiveness of traditional medicine for severe malaria and experience of high case-fatality rate in the hospital.

The trend was towards fewer fatalities after traditional treatment $(11 \%)$ than after modern treatment $(26 \%)$. One explanation might be that some of those considered as suffering from severe malaria in our sample actually had another less fatal illness (the same applies to the $2 \%$ of fatalities observed after presumed uncomplicated malaria: it might have been another disease). Uncomplicated malaria might have become severe, although in this case the patient should have been registered only once, as a severe case. Another possible explanation is a certain degree of effectiveness of some local, traditional treatments. Even if they are less effective than modern drugs in absolute terms, they could improve the prognosis of malaria patients because of the shorter time lag between onset of the malaria episode and treatment. In other words, greater effectiveness of care offered in modern health centres might be offset by the fact that patients arrive there after a long journey in a more critical state. Such a hypothesis could be tested with a non-experimental design, for ethical reasons (Willcox et al., 2004).

The plants found to have high activity against chloroquine-resistant $P$. falciparum were not necessarily described as such in the literature. Spondias mombin L. (Anacardiaceae) is a medium-sized tree bearing edible fruits ('hog plums'), common in both South America and tropical Africa. The bark and leaves are traditionally used for disinfections (e.g. abscesses, caries, conjunctivitis). Antiherpetic tannins have been isolated and characterized from this plant. Opilia celtidifolia (Guill. et Perr.) (Opiliaceae) is either a small tree or a creeper reaching $8-10 \mathrm{~m}$ and restricted to tropical West Africa. The roots and leaves are used to treat fever, influenza and intestinal worms. Rare modern studies on this plant demonstrated its high content in saponins and tannins. Argemone mexicana L. (Mexican poppy or prickly poppy) is a robust herbaceous annual, a member of the poppy family (Papaveraceae) 
(Arbonnier, 2002). This weed looks like a thistle, but its yellow flowers are characteristic of the Papaveraceae, as is its abundant bright yellow latex. Typical chemical constituents are benzophenanthridine alkaloids (among them berberine, protopine and sanguinarine). Securinega virosa (Roxb. ex Willd.) (Euphorbiaceae) is a $2-3 \mathrm{~m}$ shrub common in West Africa, but also in Australia and Asia. It has numerous traditional uses, and its roots are frequently described as soporific and analgesic, although its leaves are considered a stimulant and an aphrodisiac. This plant has been studied for trypanocidal activity.

Limitations of this study include the uncertainty created by the controversial definition of malaria in areas where diagnosis and therapeutic decisions must be performed without laboratory facilities. The fact that the diagnosis of malaria, both severe and uncomplicated, is not by necessity confirmed greatly weakens the conclusions made. Definitions of malaria by traditional practitioners probably result in over-diagnosis: in a study by Willcox et al. (unpublished) of 245 patients diagnosed with 'soumaya' (considered equivalent to uncomplicated malaria) by a traditional practitioner in south Mali, 30 had no plasmodia in their blood and 118 had only a low parasitaemia ( $<2000$ parasites/ $\mu$ l), thus not reaching commonly used criteria of malaria. Observation of actual therapeutic itineraries along categories of 'first' and 'second' treatments is very schematic and probably elicits only part of the very complex process of health-care-seeking behaviours. Data may be incomplete and biased, because they are based on memories of the past 6 months. Missing data on progress was probably due to the interviewer, because it is unlikely that parents forgot the outcome of their child's disease. For dramatic events the recall errors are probably small, but for details they can be important.

For severe malaria, recommendations are to bring the sick child to the health centre as fast as possible. However, travel is often difficult, especially during the rainy season, and health professionals attribute many of their treatment failures to delayed access to modern treatment in the case of severe malaria, as well as delayed or inappropriate treatment in the case of uncomplicated malaria (Muller et al., 2003; Noor et al., 2003). The first few malaria episodes are the most dangerous, because immunity to malaria rises rapidly after a limited number of infections (Gupta et al., 1999), so it seems important to study every potential way of improving the care of young patients.

After this study, we suggest that the potential role of traditional treatments for malaria should be further investigated. To start with, a retrospective outcome and treatment study seems very useful. Then one can search for new antimalarial molecules. But other interesting directions exist as well: the development of new 'medicaments traditionnels améliorés' (improved traditional medicines), which is a national policy in Mali; and the study of potential recommendations for home first-aid treatments with local resources. The study of physiological and immunomodulating actions of traditional antimalarials also deserves more attention: some local treatments could be found to act in a way that is not antiparasitic as such. They might counterbalance metabolic or haemodynamic disturbances that are life-threatening in malaria; some products may correct hypoglycaemia or lactic acidosis, improve capillary flow, prevent convulsions, or boost the immune response. Anecdotal reports in the course of this study, of patients waking immediately after an inhalation, may be an indication of product effect not solely related to the parasite cycle. To determine physiologic activities would require specific research (for example with animal models or healthy volunteers).

\section{Conclusions}

In the areas studied, self-medication and traditional medicines for malaria divert a high proportion of patients away from modern health centres, especially for severe episodes. For the latter, case-fatality rates tend to be lower after traditional home- or village-based treatments, although without any statistically significant difference. This suggests that distance, poor quality service, or economic barriers are not the only reasons why attendance to health centres is poor, but also perceived effectiveness of alternative care. Rather than ignoring or suppressing this situation, a pragmatic approach could be to study the potential effectiveness of some home-care and whether local or regional synergies between different health systems could improve patient outcome.

\section{Conflicts of interest statement}

The authors have no conflicts of interest concerning the work reported in this paper.

\section{Acknowledgements}

Thanks to the populations and authorities of Bandiagara and Sikasso Regions for their warm welcome and active participation in the study; André Rougemont for help in protocol design and logistics; Diafara Berthé, Olivier Martin and Swiss and Malian doctoral students and surveyors for help in data collection; Reto Brun and his team from the Swiss Tropical Institute for in-vitro tests; Bernard Burnand for constructive comments on the study results; and Merlin Willcox for suggestions on an earlier version of this paper. This study was supported by The Swiss Cooperation Agency.

\section{References}

Arbonnier, M., 2002. Arbres, arbustes et lianes des zones sèches d'Afrique de l'Ouest, second ed. CIRAD, MNHN, Paris.

Basco, L.K., 2004. Molecular epidemiology of malaria in Cameroon. XIX. Quality of antimalarial drugs used for self-medication. Am. J. Trop. Med. Hyg. 70, 245-250.

Bennett, S., Woods, T., Liyanaga, W.M., Smith, D.L., 1991. A simplified general method for cluster-sample surveys of health in developing countries. World Health Stat. Rep. 44, 98-106.

Berche, T., 1994. Anthropologie et Santé Publique en Pays Dogon. Karthala, Paris.

Delley, V., Bouvier, P., Breslow, N., Doumbo, O., Sagara, I., Diakite, M., Mauris, A., Dolo, A., Rougemont, A., 2000. What does a single determination of malaria parasite density mean? A longitudinal survey in Mali. Trop. Med. Int. Health 5, 404-412.

Dossou-Yovo, J., Amalaman, K., Carnevale, P., 2001. [Antimalarial guidelines and therapy practices of the population of Bouake Ivory Coast]. Med. Trop. (Mars.) 61, 495-499 [in French].

Graz, B., Diallo, D., Falquet, J., Willcox, M., 2005. Screening of traditional herbal medicine: First, do a retrospective study, with correlation between diverse treatments used and reported patient outcome. J. Ethnopharmacol. 101, 338-339. 
Gupta, S., Snow, R.W., Donnelly, C.A., Marsh, K., Newbold, C., 1999. Immunity to non-cerebral severe malaria is acquired after one or two infections. Nat. Med. 5, 340-343.

Huissoud, T., Cissé, H., Damiba, C., Koné, F., 2004. Evaluation du programme PASS-MS. Bureau de la Coopération Suisse. Bamako, Mali.

Muller, O., Traore, C., Becher, H., Kouyate, B., 2003. Malaria morbidity, treatment-seeking behaviour, and mortality in a cohort of young children in rural Burkina Faso. Trop. Med. Int. Health 8, 290-296.

Noor, A.M., Zurovac, D., Hay, S.I., Ochola, S.A., Snow, R.W., 2003. Defining equity in physical access to clinical services using geographical information systems as part of malaria planning and monitoring in Kenya. Trop. Med. Int. Health 8, 917-926.
Traoré, F., 1999. Evaluation de l'activité antimalarique de Glinus oppositofolius, Nauclea latifolia, Mytragyna inermis, trois plantes utilisées en médecine traditionnelle au Mali. Doctoral thesis. University of Marseille, Marseille, France.

Tumwesigire, S., Watson, S., 2002. Health seeking behavior by families of children suspected to have malaria in Kabale. Ugand. Afr. Health Sci. 2, 94-98.

Von Seidlein, L., Clarke, S., Alexander, N., Manneh, F., Doherty, T., Pinder, M., Walraven, G., Greenwood, B., 2002. Treatment uptake by individuals infected with Plasmodium falciparum in rural Gambia, West Africa. Bull. World Health Organ. 80, 790-796.

Willcox, M., Bodeker, G., Rasoanaivo, P. (Eds.), 2004. Traditional Medicinal Plants for Malaria. CRC Press, Boca Raton, USA. 